\title{
Identificación de algunos genes asociados al proceso de germinación de la conidia al micelio en Paracoccidioides brasiliensis
}

\author{
Ana María García ${ }^{1,2}$, Orville Hernández ${ }^{1,3}$, Beatriz H. Aristizábal ${ }^{4}$, Luz Elena Cano ${ }^{3,5}$, \\ Ángela Restrepo ${ }^{3}$, Juan G. McEwen ${ }^{1,6}$ \\ 1 Unidad de Biología Celular y Molecular, Corporación para Investigaciones Biológicas, Medellín, Colombia \\ 2 Facultad de Ciencias de la Salud, Universidad Pontificia Bolivariana, Medellín, Colombia \\ ${ }^{3}$ Unidad de Micología Médica y Experimental, Corporación para Investigaciones Biológicas, Medellín, \\ Colombia \\ ${ }^{4}$ Laboratorio de Biología Molecular, Hospital Pablo Tobón Uribe, Medellín, Colombia \\ ${ }^{5}$ Escuela de Microbiología, Universidad de Antioquia, Medellín, Colombia \\ ${ }^{6}$ Facultad de Medicina, Universidad de Antioquia, Medellín, Colombia
}

Introducción. Paracoccidioides brasiliensis es un hongo dimórfico térmico, que a temperatura ambiente se presenta como un moho productor de conidias, mientras que en el huésped se comporta como una levadura de gemación múltiple. Los mecanismos moleculares que rigen la germinación de conidia a micelio aún se desconocen.

Objetivo. Estudiar en $P$. brasiliensis la cinética del proceso de germinación de conidia a micelio y determinar los genes expresados durante este proceso mediante la construcción y el análisis de una librería EST (Expressed Sequence Tag).

Materiales y métodos. Para el estudio de la cinética de germinación, se produjeron y aislaron conidias de $P$. brasiliensis. Estas fueron incubadas en cultivos líquidos a $18^{\circ} \mathrm{C}$ por $24,48,72$ y 96 horas, y se examinaron por microscopía de luz. A partir de conidias cultivadas por 96 horas, se construyó y caracterizó una librería EST, la cual representaría los genes expresados durante el proceso de germinación.

Resultados. Durante el proceso de germinación de conidia a micelio, se observó $11,7 \pm 1,2 \%$, $30 \pm 0,6 \%, 43 \pm 1,3 \%$ y $66 \pm 2,4 \%$ de germinación a las $24,48,72$ y 96 horas de incubación, respectivamente. Además, se obtuvo una librería del proceso de germinación consistente en 129 secuencias agrupadas en cuatro secuencias contiguas y siete secuencias únicas, para un total de 11 posibles genes. Ocho secuencias (72,7\%) no habían sido descritas anteriormente en otras librerías informadas para este hongo y podrían representar genes específicos de la germinación de conidia a micelio.

Conclusiones. Éste es el primer reporte en el que se identifican genes no descritos anteriormente, que son expresados durante la germinación de conidia a micelio, proceso de gran importancia en la biología de $P$. brasiliensis.

Palabras clave: Paracoccidioides, esporas fúngicas, micelio, germinación.

Identification of genes associated with germination of conidia to form mycelia in the fungus Paracoccidioides brasiliensis

Introduction. Paracoccidioides brasiliensis is a thermo-dimorphic fungus. At room temperature it grows as a mold that produces conidia, whereas in the vertebrate host it grows as a multiplebudding yeast. The molecular mechanisms involved in the germination from the conidia to the mycelia process remain unknown.

Objective. The kinetics of conidia to mycelia germination process were studied in the dimorphic fungus $P$. brasiliensis. Gene expression during this process was evaluated by construction and analysis of an EST library.

Materials and methods. For the germination kinetics study, $P$. brasiliensis conidia were isolated as single cell units. Then, they were cultured at $18^{\circ} \mathrm{C}$ in BHI (brain-heart infusion) broth for 24,48 , 72 and $96 \mathrm{hr}$. After each perion, they were examined by light microscopy. From conidia harvested 
at $96 \mathrm{hr}$, an EST library was constructed; at this stage the gene expression was presumed to be maximal for the germination process.

Results. During the conidia to the mycelia developmental process, the following germination rates were observed: at $24 \mathrm{hr}, 11.7 \pm 1.2 \%$; at $48 \mathrm{hr}, 30 \pm 0.6 \%$; at $72 \mathrm{hr}, 43 \pm 1.3 \%$; and at $96 \mathrm{hr}$, $66 \pm 2.4 \%$. At the 96 hour stage, an EST library was constructed. It consisted of 129 sequences grouped in 4 contigs and 7 singlets for a total of 11 possible genes. Eight of the sequences had not been described previously in other EST libraries of this fungus.

Conclusions. New genes were identified that were expressed during the conidia to the mycelia germination process and may represent genes specific to the germination process.

Key words: Paracoccidioides; spores; fungal; mycelium, germination.

La paracoccidioidomicosis, enfermedad infecciosa endémica de áreas tropicales y subtropicales de Centroamérica y Suramérica, es causada por el hongo dimórfico térmico Paracoccidioides brasiliensis $(1,2)$. La paracoccidioidomicosis es una enfermedad crónica que afecta especialmente a hombres adultos, trabajadores del campo. La enfermedad se desarrolla cuando las partículas infecciosas (conidias o fragmentos de micelio) que se alojan en los alvéolos pulmonares del huésped, realizan el proceso de transición hacia levadura, como respuesta para la supervivencia en los tejidos $(3,4)$.

En cultivos a $37^{\circ} \mathrm{C}$ y en los tejidos mismos, $P$. brasiliensis se encuentra en forma de levadura, estructura que se reproduce por gemación múltiple que le confiere el aspecto típico de un timón de barco (2). A temperaturas entre $18^{\circ} \mathrm{C}$ y $24^{\circ} \mathrm{C}$, el hongo se desarrolla como un moho provisto de micelios delgados, ramificados y tabicados que, en medios de cultivo pobres en carbohidratos, producen diversos tipos de microconidias (2 a $5 \mu \mathrm{m}$ ), que se consideran las partículas infecciosas (4). La conidia es una estructura en reposo que, cuando encuentra un medio propicio, comienza su proceso de germinación a micelio, si se encuentra en el medio ambiente o a temperaturas menores de $26^{\circ} \mathrm{C}$, o inicia la transición a levadura, si entra en contacto con un hospedero mamífero o si es cultivada a $36^{\circ} \mathrm{C}(2)$.

\footnotetext{
Correspondencia:

Ana María García, Unidad de Biología Celular y Molecular, Corporación para Investigaciones Biológicas, Carrera 72A №. 78B-141, Medellín, Colombia.

Fax: (574) 4415514.

agarcia@cib.org.co

Recibido: 07/11/08; aceptado:26/03/09
}

La transición de micelio a levadura ha sido estudiada por diversos investigadores, y se ha logrado establecer una escala de estadios morfológicos de esta transición y permitir su cuantificación (5-8). De esta manera, el desarrollo de levaduras a partir del micelio se da desde el primer día del cambio térmico y el desarrollo de levaduras de gemación múltiple se presenta después de 5 días (8) y se alcanza el $50 \%$ de la transición a las 48 horas de iniciado el proceso (5). De igual manera, se ha estudiado la transición de levadura a micelio (5-7) y se han evaluado los cambios morfológicos que ocurren durante este proceso, los que muestran elongación de las células en gemación con muerte y desintegración de las células madre y conversión a micelio de $50 \%$ a $60 \%$ de las células, a las 48 horas del cambio térmico.

De igual manera, la transición de conidia a levadura ha sido ampliamente estudiada por diversos autores, tanto en ensayos in vivo (9-11) como ex vivo (12-15); éstos han demostrado que, en promedio, el $50 \%$ de esta transición se logra después de 48 horas de incubación a $36^{\circ} \mathrm{C}$ y llega hasta el $80 \%$ a las 72 horas. Sin embargo, es poco lo que se conoce sobre el proceso de germinación de conidia a micelio. Restrepo et al. (16) reportaron por primera vez el proceso de germinación in vitro y demostraron que la elongación de las hifas ocurría después de 60 horas de incubación a $25^{\circ} \mathrm{C}$. Posteriormente, Restrepo et al. (Restrepo BI, McEwen JG, Salazar ME, Restrepo A. The mycelial form of Paracoccidioides brasiliensis. In: $\mathrm{X}$ Congress of the International Society for Human and Animal Mycology-ISHAM; 1988. p. 143-8) confirmaron el desarrollo del proceso de germinación, indicando 
que a $25^{\circ} \mathrm{C}$ y en 96 horas, se producían los tubos germinales, los que posteriormente progresaban hasta llegar a elementos de micelios.

Con respecto a la evaluación de los mecanismos moleculares y de la expresión de genes en los diferentes estadios morfológicos, se han realizado varios estudios utilizando estrategias, tales como la construcción de librerías EST (Expressed Sequence Tag) y los microarreglos, con los que se ha logrado recopilar un gran número de genes que se expresan durante los estadios de micelio y levadura $(5,17,18)$, así como genes que presentan expresión diferencial durante la transición de micelio a levadura $(6,19)$. Estos estudios han abierto las puertas al conocimiento de los mecanismos genéticos que rigen la diferenciación morfológica de $P$. brasiliensis; sin embargo, los procesos moleculares involucrados en los cambios morfológicos de las conidias y los genes involucrados en su transición a levadura y germinación a micelio, han sido muy poco estudiados. No obstante, a partir de ARNm obtenido a las 48 horas del proceso de transición, fue posible construir una pequeña librería EST, compuesta por 79 posibles secuencias, de las cuales, 39 no fueron encontradas en otras librerías de $P$. brasiliensis y que, por lo tanto, parecen ser específicas de la transición de conidia a levadura, ya que no habían sido descritas previamente en otras librerías obtenidas a partir de este hongo (García AM, et al. En prensa, Medical Mycology).

De esta manera, en este trabajo se describe la cinética de la germinación de conidia a micelio, así como la construcción y análisis de una librería EST obtenida a partir del ARNm extraído a las 96 horas de tal proceso de germinación.

\section{Materiales y métodos}

\section{Aislamiento de $\boldsymbol{P}$. brasiliensis y mantenimiento del hongo}

Se utilizó la cepa de $P$. brasiliensis ATCC N60855, depositada en la American Type Culture Collection, provenientes de la colección de la Corporación para Investigaciones Biológicas, la cual es productora de conidias (20). La cepa fue mantenida en su fase micelial en medio sólido
Mycosel ${ }^{\circledR}$ (BD, Sparks, MD USA) y en medio sintético McVeight Morton modificado (SMVM) (21) a $18^{\circ} \mathrm{C}$ por dos a tres semanas.

\section{Producción y purificación de conidias de $P$. brasiliensis}

Para la producción de las conidias se utilizó el protocolo previamente descrito por Restrepo et al. (20) en el cual la cepa es mantenida en su fase de micelio en medio sólido para hongos SMVM (21) a $18^{\circ} \mathrm{C}$ durante dos a tres semanas para, posteriormente, transferir el correspondiente crecimiento de micelios a medio SMVM líquido e incubarlo a $18^{\circ} \mathrm{C}$ en agitación constante (150 rpm) por dos semanas. El crecimiento obtenido era homogenizado por licuado (Waring blendor) $y$ transferido a medios de cultivo pobres en carbohidratos (agar agua), con incubación a $18^{\circ} \mathrm{C}$ por un período mínimo de tres meses, tiempo necesario para la esporulación del hongo (20).

Para la obtención de las conidias, se usó la estrategia de purificación con lana de vidrio, la cual fue utilizada, tanto para realizar la cinética de la germinación como para obtener el ARNm destinado a la construcción de las librerías.

Para la purificación con lana de vidrio, se lavaron las cajas con solución salina $0,85 \%$ más Tween 20 al 0,01\%; la suspensión se transfirió a un Erlenmeyer con perlas de vidrio y se puso en agitación a 250 rpm por 45 minutos a temperatura ambiente $\left(18^{\circ} \mathrm{C}\right)$, con el fin de fragmentar el micelio y liberar las conidias. Posteriormente, la purificación se realizó pasando la suspensión de conidias por jeringas rellenas con lana de vidrio, en la cual los rastros de micelios quedaban atrapados, obteniéndose sólo las partículas más pequeñas correspondiente a las conidias (20).

Los sedimentos obtenidos con este protocolo, se lavaron dos veces con PBS y se centrifugaron a $1.500 \mathrm{~g}$ a $4^{\circ} \mathrm{C}$ por 30 minutos para, finalmente, resuspenderlos en $1 \mathrm{ml}$ de PBS del correspondiente lavado con conidias. Con ellas se preparó una dilución 1:10, con la cual se hizo recuento del número de conidias en hemocitómetro y la prueba de viabilidad por la técnica de bromuro de etidio-diacetato de fluoresceína (BE-DF) (22). 


\section{Determinación de la cinética de germinación de conidia a micelio de $P$. brasiliensis}

Esta cinética se realizó en microplatos de cultivo celular (Linbro ${ }^{\circledR}$ Tissue culture multi-well plate ICN: 7636405) con medio BHI (Brain Heart Infusion BD, USA), líquido con suplemento de glucosa al $1 \%$ y asparagina al $0,2 \%$, platos que se inocularon con 25.000 conidias por pozo, con incubación a $18^{\circ} \mathrm{C}$ y agitación constante por 24 , 48, 72 y 96 horas. En cada uno de los tiempos señalados, se realizó observación microscópica directa de los platos y se determinó la tasa de germinación basada en los cambios morfológicos descritos previamente, para evaluar la geminación con base en: i) la producción de tubo germinal, ii) la elongación de las hifas y iii) la producción de micelio (Restrepo BI, McEwen JG, Salazar ME, Restrepo A. The mycelial form of Paracoccidioides brasiliensis. In: $\mathrm{X}$ Congress of the International Society for Human and Animal Mycology - ISHAM; 1988. p.143-8) (6).

\section{Obtención de ARN total}

El ARN total se obtuvo después de exponer las conidias a inducción térmica de $18^{\circ} \mathrm{C}$ por 96 horas, rompiendo las células por maceración con nitrógeno líquido y con posterior extracción mediante la técnica del Trizol® , de acuerdo con las indicaciones del fabricante (Invitrogen Life Technologies, Pasley, CA., U.S.A, Cat: 15596-026).

\section{Amplificación de los ácidos nucleicos y obtención de ADNc}

El ARN obtenido de las conidias se purificó utilizando el juego de reactivos PicoPure ${ }^{\circledR}$ (Aracturus Mountain View, CA. USA. Cat. Kit 0202). Posteriormente, a partir de este ARN total purificado, se amplificó el ARNm utilizando el juego de reactivos RiboAmp® (Aracturus, Mountain View CA, USA. Cat. Kit 0201). El ARNm así amplificado fue transcrito a ADNc utilizando Superscript ${ }^{\circledR}$ (juego de reactivos Superscript ${ }^{\circledR}$ cloning Kit-Invitrogen Life Technologies, Pasley, CA., USA, Cat. 18248-013) y siguiendo las indicaciones del fabricante.

\section{Construcción de la librería EST}

Una vez obtenido elADNc, éste se clonó utilizando el juego de reactivos TOPO TA (Cloning ${ }^{\circledR}$ kit for sequencing, Invitrogen, Life Technologies, Pasley, CA., USA, Cat. K450030) y siguiendo las indicaciones del fabricante. Posteriormente, el material se transformó en la cepa de Escherichia coli DH5 a por electroporación. Las células transformadas se cultivaron en caldo de Luria sólido con $100 \mu \mathrm{g} / \mathrm{ml}$ de ampicilina, IPTG $(20 \mu \mathrm{g} /$ $\mathrm{ml}$ ) y Xgal $(100 \mu \mathrm{g} / \mathrm{ml})$, para confirmar la pérdida de la $\alpha$-complementación. Las colonias blancas así obtenidas se aislaron individualmente y se cultivaron a $37^{\circ} \mathrm{C}$ toda la noche en placas de 96 pozos con caldo de Luria líquido más 15\% de glicerol y $100 \mu \mathrm{g} / \mathrm{ml}$ de ampicilina para, posteriormente, ser almacenadas a $-80^{\circ} \mathrm{C}$.

\section{Secuenciación de plásmidos}

Para obtener el material destinado a secuenciación, se realizó una miniprep en placa de 96 pozos, según el protocolo descrito por Marra et al. (23). La secuenciación de los fragmentos insertados en los plásmidos así obtenidos, se realizó utilizando Big Dye®, versión 3.0 (Applied Biosystems Foster City, California USA), con el iniciador M13R, el que se utilizó como plantilla para los plásmidos ya extraídos, llevándolos a un secuenciador automático de fluorescencia ABI 3100 (ABI PRISM®), por el Grupo de Biología Molecular de la Facultad de Ciencias Farmacéuticas de la Universidad de São Pãulo, Ribeirão Preto, Brasil.

\section{Análisis de las librerías}

El análisis y ensamblaje de la secuencias de la librería EST se realizó mediante un pipeline programado por el Grupo de Biología Molecular de la Facultad de Ciencias Farmacéuticas de la Universidad de São Pãulo, Ribeirão Preto, Brasil, mediante el cual se editaban las secuencias automáticamente, limpiándolas de las secuencias no deseadas, como son las secuencias del vector, ARNr de hongos y secuencias correspondientes a bacterias. Posteriormente, el programa agrupó las secuencias (clustering) para obtener las secuencias contiguas (contigs) y las secuencias únicas (singlets). El pipeline se construyó por medio de los programas Phrap (24) and Cap3 (25). Una vez depurada la información de las secuencias obtenidas a partir de la librería, aquéllas se evaluaron contra diferentes bases 
de datos, buscando la similitud con secuencias de genes y proteínas previamente reportadas. Para este análisis se utilizaron los programas BlastN y BlastX con las bases de datos de GenBank (http://www.ncbi.nlm.nih.gov/BLAST/), EMB -Fungal EST (http://www.ebi.ac.uk/ blast2/nucleotide.html) y la base de los EST de levaduras publicados por Goldman et al. (http://143.107.203.68/pbver2/default.html) (5).

La validez de la identidad de las secuencias se categorizó con base en el valor de $E$ (probabilidad de error) obtenido en los resultados del Blast. Se obtuvo, también, el porcentaje de secuencias según su categoría funcional. Las categorías funcionales establecidas fueron: respuesta al estrés, señalización intracelular, metabolismo, estructura, proteína hipotética conservada y proteína hipotética. Se evaluó, además, el porcentaje de secuencias presentes en las librerías EST de Goldman y Felipe en 2003, mediante la la base de datos Fungi-EST del EMBL, con el fin de determinar aquéllas que, por no estar presentes en estas librerías, podían considerarse secuencias específicas del proceso de germinación de conidia a micelio $(5,17,26)$.

\section{Resultados}

\section{Cinética de la germinación}

Durante el seguimiento de la cinética de la germinación, se observó que $11,7 \pm 1,2 \%$ de las conidias habían producido tubos germinales o hifas cortas a las 24 horas de incubación a $18^{\circ} \mathrm{C}$. Posteriormente, después de las 48 horas, la proporción de células germinadas aumentaba a $30 \pm 0,6 \%$, anotándose un crecimiento paralelo en la longitud de las hifas; fue, entonces, que las primeras hifas ramificadas se hicieron evidentes, aunque en baja proporción. A las 72 horas, el $43 \pm 1,3 \%$ de las conidias presentaban ya germinación, con un aumento en la presencia de hifas ramificadas. Finalmente, a las 96 horas, el $66 \pm 2,4$ de las conidias había germinado y se observaban hifas largas ramificadas (figura 1).

\section{Construcción y análisis de la librería}

A partir del ARNm de conidias cultivadas a $18^{\circ} \mathrm{C}$ por 96 horas, se obtuvo una librería compuesta por 198 colonias, las que se conservaron a $-80^{\circ} \mathrm{C}$. Después de la extracción de plásmidos, la secuenciación de los insertos y del paso de las secuencias obtenidas por el pipeline, se obtuvieron 129 secuencias, las cuales

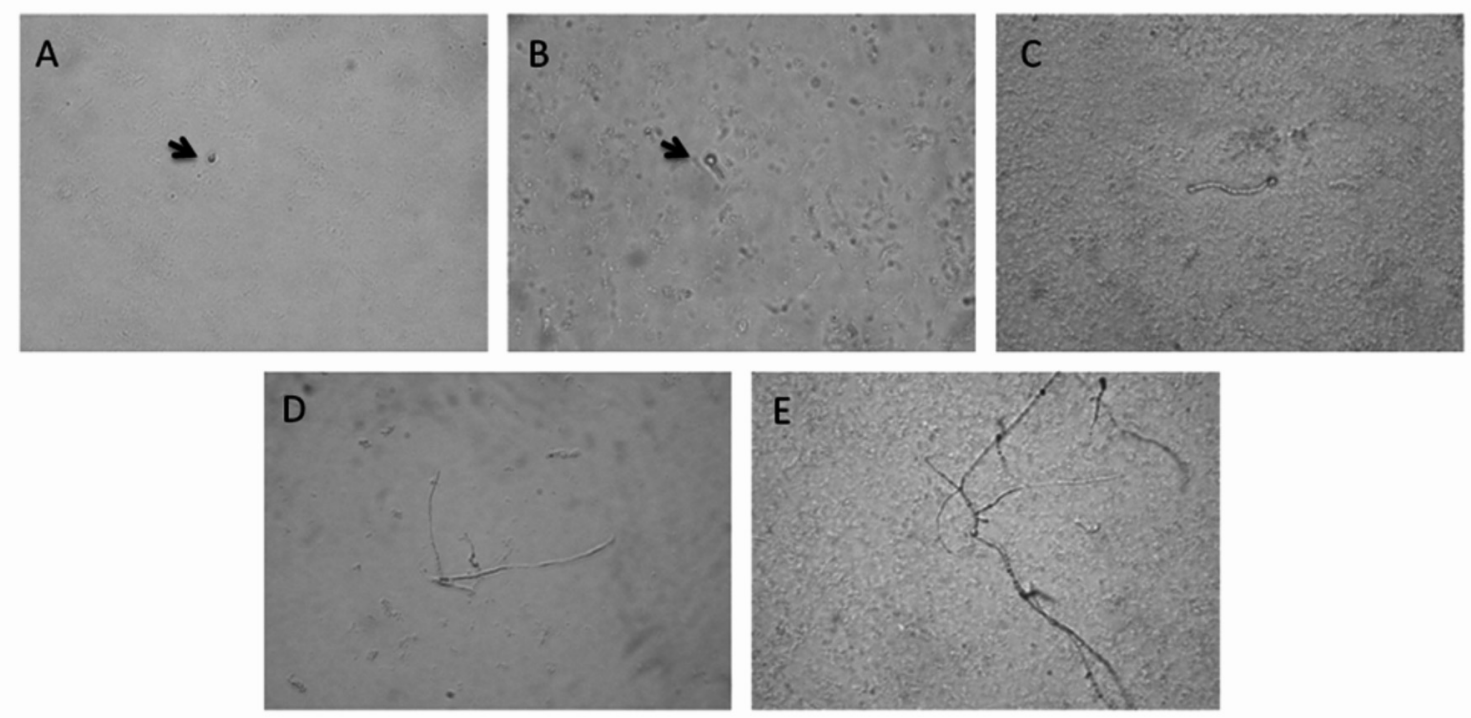

Figura 1. Diferenciación morfológica de la germinación de conidia a micelio en $P$. brasiliensis por microscopía de luz (40X). A) 0 horas de cultivo. B) 24 horas de cultivo, se observan tubos germinales. C) 48 horas de cultivo, aumento de la longitud de los tubos germinales. D y E) 72 y 96 horas de cultivo se observan hifas ramificadas. 
se agruparon en siete secuencias contiguas y siete secuencias únicas, para un total de 14 posibles secuencias codificantes para proteínas relacionadas con la germinación de conidia a micelio. Durante el análisis Blast, se descartaron cuatro secuencias adicionales, dada su alta similitud con secuencias ribosómicas y mitocondriales (secuencias contiguas 1, 3 y 4), dejando 11 posibles genes, 4 secuencias contiguas y 5 secuencias únicas que correspondieron a proteína hipotética o proteína hipotética conservada (códigos de acceso en GenBank, http:// www.ncbi.nlm.nih.gov). Las características más importantes de las secuencias contiguas y las secuencias únicas se describen en el cuadro 1.

Al comparar la librería de germinación de conidia a micelio con las librerías EST obtenidas por Goldman et al. (5) y Felipe et al. (17), se encontró que 4 de las 11 posibles secuencias estaban presentes en estas librerías, 3 de ellas en la descrita por Goldman et al. y una en ambas (cuadro 2). Además, al comparar nuestros resultados con los obtenidos por otros investigadores, como Nunes et al. (6), Tavares et al. (27), Bailao et al. (28) y Bastos et al. (19), no fue posible encontrar similitudes en las secuencias descritas por ellos y las nuestras.

Se evidenció, asimismo, nueva información al analizar las secuencias completas reportadas en GenBank halladas por el análisis BLAST, que poseían homología con los clones de la librería. Este análisis se hizo nuevamente por BLAST en el NCBI. De esta manera, la proteína hipotética de Podospora anserina correspondiente al contig 02, presentó similitud con una proteína RcaA de respuesta al daño del ADN (DNA damage response protein $R c a A$ ), la proteína hipotética de Aspergillus nidulans correspondiente al contig 06 presentó una similitud significativa con una proteína putativa formadora de filamentos, Tpr/p270 (Filament-forming protein putative, Tpr/p270) y la proteína hipotética conservada de Aspergillus fumigatus correpondiente al singlet 02 presentó gran similitud con la proteína ribosómica S15 (cuadro 3)

\section{Discusión}

La transición de conidia a levadura ha sido bien documentada por diversos autores; no así el proceso de germinación de conidia a micelio que sólo fue estudiado por Restrepo et al. (Restrepo BI, McEwen JG, Salazar ME, Restrepo A. The mycelial form of Paracoccidioides brasiliensis. In: $X$ Congress of the International Society for Human and Animal Mycology - ISHAM; 1988. p. 143-8) al describirlo por primera vez. Los resultados de este primer estudio se correlacionan adecuadamente con los obtenidos en este documento, en el cual se presentaron tiempos similares de evolución a los descritos previamente.

Cuadro 1. Caracterización de las secuencias expresadas durante la germinación de conidia a micelio en $P$. brasiliensis.

\begin{tabular}{|c|c|c|c|c|c|c|}
\hline \multirow[t]{2}{*}{ Conglomerado } & \multirow[t]{2}{*}{ Función } & \multirow[t]{2}{*}{ Posible proteína } & \multicolumn{4}{|c|}{ Análisis BLAST } \\
\hline & & & Organismo & I & $E$ & BLAST \\
\hline Contig 02 & Desconocida & $\mathrm{PHC}$ & Podospora anserina & 39 & $4 e-06$ & $x$ \\
\hline Contig 05 & Desconocida & $\mathrm{PHC}$ & Kluyveromyces lactis & 100 & $3 e-22$ & $x$ \\
\hline Contig 06 & Desconocida & $\mathrm{PHC}$ & Aspergillus nidulans & 39 & $2 e-21$ & $\mathrm{X}$ \\
\hline Contig 07 & Desconocida & $\mathrm{PH}$ & - & - & - & - \\
\hline Singlet 01 & Desconocida & $\mathrm{PH}$ & - & - & - & - \\
\hline Singlet 02 & Desconocida & $\mathrm{PHC}$ & Aspergillus fumigatus & 72 & $1 e-03$ & $x$ \\
\hline Singlet 03 & Desconocida & $\mathrm{PH}$ & - & - & - & - \\
\hline Singlet 04 & Desconocida & $\mathrm{PH}$ & - & - & - & - \\
\hline Singlet 05 & Desconocida & $\mathrm{PH}$ & - & - & - & - \\
\hline Singlet 06 & Desconocida & $\mathrm{PH}$ & - & - & - & - \\
\hline Singlet 07 & Desconocida & $\mathrm{PH}$ & - & - & - & - \\
\hline
\end{tabular}

I: porcentaje de identidad; E: probabilidad de error; PHC: proteína hipotética; PH: proteína hipotética conservada. La caracterización de las secuencias se realizó usando el análisis BLAST en la base de datos nr en el NCBI del GeneBank (http://blast.ncbi.nlm.nih.gov/Blast.cgi) 
Cuadro 2. Secuencias de la librería de $P$. brasiliensis durante el proceso de germinación de conidia a micelio que comparten similitud con las descritas en las bibliotecas de micelios y levadura descritas por Goldman et al. en 2003 y Felipe et al. en 2003.

\begin{tabular}{|c|c|c|c|c|c|}
\hline \multirow[t]{2}{*}{ Conglomerado } & \multirow[t]{2}{*}{ Función } & \multirow[t]{2}{*}{ Posible proteína } & \multicolumn{3}{|c|}{ BLAST en EMBL- Fungi EST } \\
\hline & & & $\mathbf{I}$ & $\mathbf{E}$ & Biblioteca en la que se identificó \\
\hline Singlet 06 & Desconocida & $\mathrm{PH}$ & 93 & $8.5 e-20$ & Goldman, 2003 \\
\hline Singlet 07 & Desconocida & $\mathrm{PH}$ & 97 & $6.7 e-30$ & Goldman, 2003 \\
\hline Singlet 02 & Desconocida & $\mathrm{PHC}$ & 95 & $3.4 e-51$ & Goldman, 2003 \\
\hline \multirow[t]{2}{*}{ Singlet 05} & Desconocida & $\mathrm{PH}$ & 98 & $8.5 e-28$ & Goldman, 2003 \\
\hline & & & 96 & $1.3 e-26$ & Felipe, 2003 \\
\hline
\end{tabular}

I: porcentaje de identidad; E: probabilidad de error; PHC: proteína hipotética; PH: proteína hipotética conservada. La caracterización de las secuencias se realizó usando el análisis BLAST en la base de datos "Fungi-EST data bases" del EMBL (http://www.ebi.ac.uk /blast2/nucleotide.html).

Cuadro 3. Secuencias informativas de la librería de $P$. brasiliensis durante el proceso de germinación de conidia a micelio.

\begin{tabular}{|c|c|c|c|c|c|}
\hline \multirow[t]{2}{*}{ Conglomerado } & \multirow[t]{2}{*}{ Función } & \multirow[t]{2}{*}{ Posible proteína } & \multicolumn{3}{|c|}{ Análisis BLAST } \\
\hline & & & Organismo & $I$ & $E$ \\
\hline $\begin{array}{l}\text { PCH de Podospora anserina } \\
\text { (Contig 02) }\end{array}$ & Ciclo celular & $\begin{array}{l}\text { Proteína de respuesta } \\
\text { al daño RcaA }\end{array}$ & $\begin{array}{l}\text { Neosartorya } \\
\text { fischeri }\end{array}$ & 48 & $1 e-165$ \\
\hline $\begin{array}{l}\text { PHC de Aspergillus nidulans } \\
\text { (Contig 06) }\end{array}$ & Estructural & $\begin{array}{l}\text { Proteína formadora } \\
\text { de filamentos- } \\
\text { (Tpr/p270), putativa }\end{array}$ & $\begin{array}{l}\text { Neosartorya } \\
\text { fischeri }\end{array}$ & 64 & 0.0 \\
\hline $\begin{array}{l}\text { PHC de Aspergillus fumigatus } \\
\text { (Singlet 02) }\end{array}$ & $\begin{array}{l}\text { Síntesis } \\
\text { proteica }\end{array}$ & $\begin{array}{l}\text { Proteína ribosómica } \\
\text { S15, putativa }\end{array}$ & $\begin{array}{l}\text { Neosartorya } \\
\text { fischeri }\end{array}$ & 54 & $3 e-60$ \\
\hline
\end{tabular}

I: porcentaje de identidad; E: probabilidad de error. La caracterización de las secuencias se realizó usando el análisis BLAST en la base de datos nr en el NCBI del GeneBank (http://blast.ncbi.nlm.nih.gov/Blast.cgi)

El fenómeno de transición de levadura a micelio también se ha estudiado pero, al igual que en el caso de la germinación de conidia a micelio, no tan exhaustivamente. Se han descrito algunas características de este proceso en el cual, a las 48 horas, $50 \%$ a $60 \%$ de las levaduras han producido ya tubos germinales o primordios de hifas, señal clara del cambio morfológico (5-7,29). Esta transición ocurre de una manera más rápida a lo observado en la de conidia a micelio, en donde sólo se logra un $66 \%$ de germinación a las 96 horas.

Con respecto a los genes que estuvieran relacionados con el proceso de germinación de conidia a micelio, nada se ha descrito hasta el momento, ya que el interés siempre se ha centrado en la expresión de genes durante la transición de conidia a levadura, dada su importancia en el proceso patogénico, mientras que los mecanismos relacionados con la permanencia del hongo en la naturaleza se encuentran sin estudiar. Ésta es la primera vez que se describen secuencias génicas posiblemente relacionadas con el proceso de germinación de conidia a micelio en $P$. brasiliensis.

De las secuencias descritas en esta librería, tres se encontraban presentes en la de levaduras descrita por Goldman et al. (5) y una estaba presente en las de Felipe et al. (17) y Goldman et al. (5), lo que demuestra su participación en los estadios morfológicos de micelio, de levadura o de ambos. Las siete secuencias restantes no habían sido descritas anteriormente, por lo cual pueden considerarse como secuencias específicas del proceso de germinación de conidia a micelio. Sin embargo, debe tenerse en cuenta que estas secuencias podrían corresponder a regiones génicas diferentes ya descritas, pero que no detectan secuencias similares dentro las librerías EST dada su naturaleza, ya que las EST 
corresponden, usualmente, sólo a secuencias parciales de los genes, hecho que debe ser verificado con análisis adicionales.

Además, cuatro secuencias se clasificaron como proteínas hipotéticas conservadas (posibles proteínas descritas en otros organismos, pero de función desconocida) y siete fueron proteínas hipotéticas (no descritas previamente en otros organismos). Asimismo, no se encontró ninguna similitud entre estos resultados y los obtenidos en otros estudios $(6,19,27,28)$.

Un análisis posterior de estos genes mostró algunos dominios que confieren función conocida a tres de las secuencias halladas. El contig 02 presentó dominios con homología con una proteína RcaA, de respuesta al daño del ADN (DNA damage response protein-RcaA). Esta proteína ha sido descrita en $A$. fumigatus, A. clavatus, Neosartorya fischeri (30) y Pyrenophora triticirepentis (http://kr.expasy.org/ uniprot/B2WAW4). En levaduras, las proteínas que regulan la respuesta al daño del ADN, tal como lo hace RcaA, también se necesitan para mantener la longitud de los telómeros, cumpliendo así funciones protectoras para la integridad del ADN (31). Su implicación en el proceso de germinación no es claro, por lo que serían necesarios nuevos estudios para definir su importancia.

El contig 06 presentó importante similitud con una proteína putativa formadora de filamentos, Tpr/ p270 (filament-forming roteinputative, Tpr/p270). Esta proteína ha sido descrita en $A$. clavatus, $A$. fumigatus y $N$. fischeri (http://kr.expasy.org/cgibin/sprot-search-de?\%22Filamentforming\%20 protein\%20putative\%20Tpr\%2Fp270\%22). Ésta hace parte de los poros de la membrana nuclear y parece estar involucrada de diversas maneras con el ensamblaje nuclear, la importación y el transporte de proteínas del citoplasma al núcleo (32). Su función como transportadora de proteínas del citoplasma al núcleo puede reflejar la fase final de los procesos de transdución de señales, en los que los factores de trascripción se encargan de llevar a la expresión de nuevas proteínas necesarias para el cambio morfológico que representa la germinación.
El singlet 02, además de haber sido descrito por Goldman et al. (5) en su librería de levaduras, presentó gran similitud con la proteína ribosómica S15. La proteína S15 es un componente característico de los ribosomas de eucariotas $80 \mathrm{~S}$ y se ubica en la subunidad 40, muy cerca del sitio donde se posiciona el codón del ARNm durante la traducción, lo que señala una interacción directa en este proceso (33). Esta proteína se ha descrito en Ajellomyces capsulata, Botryotinia fuckeliana, Chaetomium globosum y Magnaporthe grisea (http://kr.expasy.org/cgi-bin/sprot-searchde?ribosomal\%20S15\%20fungus), y tiene una función básica, ya que está presente en todos los estadios de desarrollo durante la síntesis proteica. Sin embargo, puede tener una mayor expresión durante la geminación, al ser inducida por la presión del cambio morfológico que lleva a la creación de nuevas estructuras.

Aunque éste es el primer estudio que reporta genes relacionados con la germinación de conidia a micelio, la información obtenida es limitada por diversas causas. Una de ellas es el tiempo requerido para la producción de las conidias del hongo, proceso que toma un mínimo de tres meses; además, el número de conidias obtenidas durante este proceso es limitado y, en el mejor de los casos, no supera los cuatro a cinco millones por caja. Otra es el gran número de conidias que se necesitan para obtener cantidades adecuadas del ARN destinado a la construcción de las librerías (no menos de 250 millones de conidias). Además, debe tenerse en cuenta que las conidias son propágulas en estado de reposo, con un metabolismo basal caracterizado por mínima expresión génica, posible causa de la poca obtención de ARN. Además, el bajo número de clones y posibles genes, bien pudieran representar aquellos genes que son específicos y están involucrados en el proceso de germinación. Sin embargo, es muy posible que esta librería no represente todas las funciones celulares involucradas en tal proceso, siendo mucho lo que falta por conocer de él.

De esta manera, se hace necesario el desarrollo de nuevos estudios en busca de otros genes relacionados con tal proceso, tales como la 
construcción de nuevas librerías y la continuación del estudio de los genes hallados en este trabajo, incluyendo la valoración de la expresión de dichos genes en los diferentes estadios morfológicos, así como la evaluación de su función utilizando sistemas de ARN de interferencia (ARNi) o ARN antisentido que regulen en el hongo la expresión de estos genes.

\section{Agradecimientos}

A todos en la Corporación para Investigaciones Biológicas que, de alguna manera, colaboraron en este trabajo. A Gustavo Goldman y a su grupo de trabajo del Laboratorio de Biología Molecular de la Facultad de Ciencias Farmacéuticas de la Universidad de São Pãulo, Ribeirão Preto, Brasil, por su colaboración en la secuenciación y análisis de la librería.

\section{Conflictos de intereses}

Los autores declaran no tener ningún conflicto de intereses.

\section{Financiación}

Este trabajo fue financiado por Colciencias, proyecto aprobado No. 41522130412669 con contrato No. 476-2002, y por el Centro para Investigación y Desarrollo-CIDI de la Universidad Pontificia Bolivariana, Medellín, registro 7918. Ana María García recibió apoyo del Programa de Apoyo a Doctorados Nacionales de Colciencias y de la Asociación Colombiana de Infectología, Capítulo de Antioquia.

\section{Referencias}

1. Brummer E, Castaneda E, Restrepo A. Paracoccidioidomycosis: an update. Clin Microbiol Rev. 1993;6:89-117.

2. Restrepo A, Tobon AM. Chapter 266. Paracoccidioides brasiliensis. In: Mandell GL, Bennetts JE, Dollin, R, editors. Principles and practice of infectious diseases. 6th edition. Philadelphia, P.A: Elsevier; 2005. p. 3062-8.

3. Camilo J, Tabares AM, Gómez BL, Aristizábal BE, Cock AM, Restrepo A. The oral route in the pathogenesis of paracoccidioidomycosis: an experimental study in $\mathrm{BALB} / \mathrm{c}$ mice infected with $P$. brasiliensis conidia. Mycopathologia. 2001;151:57-62.

4. McEwen JG, Brummer E, Stevens DA, Restrepo A. Effect of murine polymorphonuclear leukocytes on the yeast form of Paracoccidioides brasiliensis. Am J Trop Med Hyg. 1987;36:603-8.
5. Goldman GH, dos Reis E, Duarte DC, de Souza LA, Quiapin AC, Vitorelli PM, et al. Expressed sequence tag analysis of the human pathogen Paracoccidioides brasiliensis yeast phase: identification of putative homologues of Candida albicans virulence and pathogenicity genes. Eukaryot Cell. 2003;2:34-48.

6. Nunes LR, Costa de Oliveira R, Leite DB, da Silva VS, dos Reis E, da Silva ME, et al. Transcriptome analysis of Paracoccidioides brasiliensis cells undergoing myceliumto-yeast transition. Eukaryot Cell. 2005;4:2115-28.

7. Queiroz-Telles F. Paracoccidioides brasiliensis: Ultrastructural findings. In: Franco LC, Restrepo A, Del Negro G, editors. Paracoccidioidomycosis. Boca Raton, FL: CRC Press; 1994. p. 27-48.

8. Salazar ME, Restrepo A. Morphogenesis of the mycelium-to-yeast transformation in Paracoccidioides brasiliensis. Sabouraudia. 1985;23:7-11.

9. Aristizábal BH, Clemons KV, Stevens DA, Restrepo A. Morphological transition of Paracoccidioides brasiliensis conidia to yeast cells: in vivo inhibition in females. Infect Immun. 1998;66:5587-91.

10. Cock AM, Cano LE, Vélez D, Aristizábal BH, Trujillo J, Restrepo A. Fibrotic sequelae in pulmonary paracoccidioidomycosis: histopathological aspects in BALB/c mice infected with viable and non-viable Paracoccidioides brasiliensis propagules. Rev Inst Med Trop Sao Paulo. 2000;42:59-66.

11. McEwen JG, Bedoya V, Patino MM, Salazar ME, RestrepoA. Experimentalmurineparacoccidiodomycosis induced by the inhalation of conidia. J Med Vet Mycol. 1987;25:165-75.

12. Cano LE, Brummer E, Stevens DA, Restrepo A. An evaluation of the enzyme-linked immunoabsorbent assay (ELISA) for quantitation of antibodies to Paracoccidioides brasiliensis. J Med Vet Mycol. 1986;24:467-75.

13. Cano LE, Gómez B, Brummer E, Restrepo A, Stevens DA. Inhibitory effect of deferoxamine or macrophage activation on transformation of Paracoccidioides brasiliensis conidia ingested by macrophages: reversal by holotransferrin. Infect Immun. 1994;62:1494-6.

14. González A, Aristizábal BH, Gómez EC, Restrepo A, Cano LE. Short report: Inhibition by tumor necrosis factor-alpha-activated macrophages of the transition of Paracoccidioides brasiliensis conidia to yeast cells through a mechanism independent of nitric oxide. Am J Trop Med Hyg. 2004;71:828-30.

15. González A, de Gregori W, Vélez D, Restrepo A, Cano LE. Nitric oxide participation in the fungicidal mechanism of gamma interferon-activated murine macrophages against Paracoccidioides brasiliensis conidia. Infect Immun. 2000;68:2546-52.

16. Restrepo BI, McEwen JG, Salazar ME, Restrepo A. Morphological development of the conidia produced by Paracoccidioides brasiliensis mycelial form. J Med Vet Mycol. 1986;24:337-9. 
17. Felipe MS, Andrade RV, Petrofeza SS, Maranhao AQ, Torres FA, Albuquerque $\mathrm{P}$, et al. Transcriptome characterization of the dimorphic and pathogenic fungus Paracoccidioides brasiliensis by EST analysis. Yeast. 2003;20:263-71.

18. Felipe MS, Torres FA, Maranhao AQ, SilvaPereira I, Pocas-Fonseca MJ, Campos EG, et al. Functional genome of the human pathogenic fungus Paracoccidioides brasiliensis. FEMS Immunol Med Microbiol. 2005;45:369-81.

19. Bastos KP, Bailao AM, Borges CL, Faria FP, Felipe MS, Silva MG, et al. The transcriptome analysis of early morphogenesis in Paracoccidioides brasiliensis mycelium reveals novel and induced genes potentially associated to the dimorphic process. BMC Microbiol. 007;7:29.

20. Restrepo A, Salazar ME, Cano LE, Patiño MM. A technique to collect and dislodge conidia produced by Paracoccidioides brasiliensis mycelial form. J Med Vet Mycol.1986;24:247-50.

21. Restrepo A, Jiménez BE. Growth of Paracoccidioides brasiliensis yeast phase in a chemically defined culture medium. J Clin Microbiol.1980;12:279-81.

22. Calich VL,PurchioA,PaulaCR.Anewfluorescentviability test for fungi cells. Mycopathologia.1979;66:175-7.

23. Marra MA, Kucaba TA, Hillier LW, Waterston RH. High-throughput plasmid DNA purification for 3 cents per sample. Nucleic Acids Res.1999;27:e37.

24. Ewing B, Green P. Base-calling of automated sequencer traces using phred. II. Error probabilities. Genome Res.1998;8:186-94.
25. Huang X, Madan A. CAP3: A DNA sequence assembly program. Genome Res.1999;9:868-77.

26. White $\mathbf{O}$, Kerlavage AR. TDB: new databases for biological discovery. Methods Enzymol.1996;266:27-40.

27. Tavares AH, Silva SS, Dantas A, Campos EG, Andrade RV, Maranhao AQ, et al. Early transcriptional response of Paracoccidioides brasiliensis upon internalization by murine macrophages. Microbes Infect.2007;9:583-90.

28. Bailao AM, Shrank A, Borges CL, Parente JA, Dutra V, Felipe MS, et al. The transcriptional profile of Paracoccidioides brasiliensis yeast cells is influenced by human plasma. FEMS Immunol Med Microbiol. 2007;51:43-57.

29. Ramírez JR. Paracoccidioides brasiliensis: conversion of yeastlike forms into mycelia in submerged culture. J Bacteriol. 1971;105:523-6.

30. Fedorova ND, Khaldi N, Joardar VS, Maiti R, Amedeo $\mathrm{P}$, Anderson MJ, et al. Genomic islands in the pathogenic filamentous fungus Aspergillus fumigatus. PLoS Genet. 2008;4:e1000046.

31. Hand RA, Jia N, Bard M, Craven RJ. Saccharomyces cerevisiae Dap1p, a novel DNA damage response protein related to the mammalian membrane-associated progesterone receptor. Eukaryot Cell. 2003;2:306-17.

32. Gant TM, Wilson KL. Nuclear assembly. Annu Rev Cell Dev Biol. 1997;13:669-95.

33. Tranque P, Hu MC, Edelman GM, Mauro VP. rRNA complementarity within mRNAs: a possible basis for mRNA-ribosome interactions and translational control. Proc Natl Acad Sci USA. 1998;95:12238-43. 